\title{
Molecular changes during extended neoadjuvant letrozole treatment of breast cancer: distinguishing acquired resistance from dormant tumours
}

Cigdem Selli ${ }^{1,2}$, Arran K. Turnbull ${ }^{1,3}$, Dominic A. Pearce ${ }^{1}$, Ang Lii ${ }^{1}$ Anu Fernando ${ }^{1,3}$, Jimi Wills ${ }^{4}$, Lorna Renshaw ${ }^{3}$, Jeremy S. Thomas ${ }^{3}$, J. Michael Dixon ${ }^{3}$ and Andrew H. Sims ${ }^{1 *}$ (D)

\begin{abstract}
Background: The risk of recurrence for endocrine-treated breast cancer patients persists for many years or even decades following surgery and apparently successful adjuvant therapy. This period of dormancy and acquired resistance is inherently difficult to investigate; previous efforts have been limited to in-vitro or in-vivo approaches. In this study, sequential tumour samples from patients receiving extended neoadjuvant aromatase inhibitor therapy were characterised as a novel clinical model.

Methods: Consecutive tumour samples from 62 patients undergoing extended (4-45 months) neoadjuvant aromatase inhibitor therapy with letrozole were subjected to transcriptomic and proteomic analysis, representing before $(\leq 0)$, early (13-120 days), and long-term (> 120 days) neoadjuvant aromatase inhibitor therapy with letrozole. Patients with at least a $40 \%$ initial reduction in tumour size by 4 months of treatment were included. Of these, 42 patients with no subsequent progression were classified as "dormant", and the remaining 20 patients as "acquired resistant".

Results: Changes in gene expression in dormant tumours begin early and become more pronounced at later time points. Therapy-induced changes in resistant tumours were common features of treatment, rather than being specific to the resistant phenotype. Comparative analysis of long-term treated dormant and resistant tumours highlighted changes in epigenetics pathways including DNA methylation and histone acetylation. The DNA methylation marks 5methylcytosine and 5-hydroxymethylcytosine were significantly reduced in resistant tumours compared with dormant tissues after extended letrozole treatment.

Conclusions: This is the first patient-matched gene expression study investigating long-term aromatase inhibitorinduced dormancy and acquired resistance in breast cancer. Dormant tumours continue to change during treatment whereas acquired resistant tumours more closely resemble their diagnostic samples. Global loss of DNA methylation was observed in resistant tumours under extended treatment. Epigenetic alterations may lead to escape from dormancy and drive acquired resistance in a subset of patients, supporting a potential role for therapy targeted at these epigenetic alterations in the management of resistance to oestrogen deprivation therapy.
\end{abstract}

Keywords: Dormancy, Oestrogen deprivation therapy, Epigenetics, Letrozole, Sequential samples, Resistance, Microarray, Proteomics

\footnotetext{
*Correspondence: andrew.sims@ed.ac.uk

${ }^{1}$ Applied Bioinformatics of Cancer, University of Edinburgh Cancer Research

UK Centre, MRC Institute of Genetics and Molecular Medicine, Edinburgh, UK

Full list of author information is available at the end of the article
}

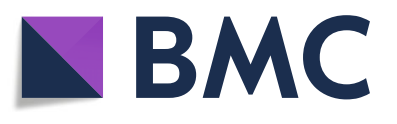

(c) The Author(s). 2019 Open Access This article is distributed under the terms of the Creative Commons Attribution 4.0 International License (http://creativecommons.org/licenses/by/4.0/), which permits unrestricted use, distribution, and reproduction in any medium, provided you give appropriate credit to the original author(s) and the source, provide a link to the Creative Commons license, and indicate if changes were made. The Creative Commons Public Domain Dedication waiver (http://creativecommons.org/publicdomain/zero/1.0/) applies to the data made available in this article, unless otherwise stated. 


\section{Background}

Approximately $70 \%$ of breast cancer patients who have oestrogen receptor (ER) alpha-positive tumours receive adjuvant oestrogen deprivation therapy. Five years of aromatase inhibitor therapy produces a $40 \%$ reduction in 10-year mortality [1]. However, while the annual risk of mortality for ER-negative breast cancer decreases following the first 5 years after diagnosis, the annual rate remains constant for $\mathrm{ER}^{+}$patients [2]. In fact, women with $\mathrm{ER}^{+}$early-stage disease treated with 5 years of adjuvant endocrine therapy have a persistent risk of recurrence and death from breast cancer for at least 20 years after diagnosis [3]. Molecular studies have demonstrated that nodal and distant metastases are highly similar to their matched primary tumours, implicating a continuation of the original cancer [4-6]. However, the time between treatment and recurrence is often greater than that which can be explained by normal cell-doubling rates [7], implying cancer cells remain dormant in the body before re-awakening.

Residual dormant cancer cells are hypothesised to persist either by withdrawing from the cell cycle and transitioning to a quiescence state or by continuing to proliferate at a reduced rate, counter-balanced by cell death [8]. Reawakened dormant cells may become detectable after reaching a detection threshold or reactivated via increased angiogenesis, and/or escape from the inhibitory microenvironment or immune effects $[9,10]$. Dormancy is therefore considered a major mechanism underlying resistance to therapy, where dormant cells survive despite anti-proliferative oestrogen deprivation therapy.

Resistance to oestrogen deprivation therapy may occur at disease inception (de novo or innate resistance), but a larger proportion of patients acquire resistance during treatment (acquired/secondary resistance) [11]. Several mechanisms of resistance to oestrogen deprivation therapy have been described previously $[12,13]$. However, the majority of these findings are based on preclinical data obtained from cell lines and animal models. It is therefore difficult to know if these accurately reflect molecular changes in patient tumours.

Expression profiling of clinical samples, measuring the effect of, or predicting response to, treatment has recently become feasible. However, experimental design issues, such as the difficulty in obtaining paired samples for comparison, particularly for longer time intervals, makes it difficult to study changes within tumours [14]. For example, a previous study investigating tamoxifen failure compared samples from patients requiring salvage surgery with pre-treatment samples from an unrelated group of disease-free patients [15]. More recently, sequential patient-matched samples have been successfully utilised to determine treatment-induced dynamic changes in tumours at 2 weeks to 3 months, demonstrating the effectiveness of this approach [16-18].

For a variety of reasons, including being unfit for surgery, a proportion of patients receiving pre-surgical oestrogen deprivation therapy do not have their tumours excised following 3-4 months of treatment. These long-term endocrine-treated tumours represent a unique group that can inform how tumours respond to extended oestrogen deprivation in situ. Having initially shrunk in size, some tumours remain at a steady volume and appear dormant, whilst others subsequently begin to regrow. We have utilised this unique cohort of sequential samples from patients receiving extended neoadjuvant oestrogen deprivation therapy to characterise luminal breast cancer dormancy and acquired resistance as a novel clinical model.

\section{Methods \\ Patients and samples}

Breast cancer patients were treated with neoadjuvant aromatase inhibitor therapy with letrozole (Femara, 2.5 mg; Novartis Pharma AG, Basel, Switzerland) for a minimum of 4 months; tumours were not removed either because patients declined or were unfit for surgery. The study was approved by the local regional ethics committee (07/S1103/26, August 2007) and all patients gave informed consent. Clinical characteristics of the tumours are given in Table 1. A consort diagram detailing inclusion and exclusion criteria is provided in Additional file 1 (Figure S1). Patients with $>40 \%$ initial decrease in tumour size by 4 months of treatment were included in the study. Those with no subsequent progression on imaging by the latest biopsy were classified as "dormant"; otherwise, they were classified as "acquired resistant" (Fig. 1a, b). For patients whose latest ultrasound scan (USS) measurement was taken more than a month before surgery, changes in gene expression (mean relative change) of three widely used proliferation markers (MKI67, PCNA, and MCM2) were used to assist classification. Tumours with an increase in proliferation marker expression (either after an initial decrease or not) were classified as "acquired resistant", otherwise there were classified as "dormant". Sequential tumour biopsies were taken with a 14-gauge needle before and after letrozole treatment and the time of surgery. Fresh samples were snap-frozen in liquid nitrogen and each tumour sample was confirmed to contain $\geq 50 \%$ cellularity and at least $60 \%$ tumour tissue using haematoxylin and eosin (H\&E) sections. Following pulverisation of tissue with a membrane disruptor (Micro-Dismembrator U, Braun Biotech), phase separation was performed by guanidinium thiocyanate-phenol-chloroform extraction (Qiazol Lysis Reagent). 
Table 1 Patient characteristics

\begin{tabular}{|c|c|c|c|c|}
\hline & Dormant, $n(\%)$ & Resistant, $n$ (\%) & Total, $n$ & $p$ value $^{a}$ \\
\hline Total no. of patients & 42 & 20 & 62 & \\
\hline Total no. of samples & 111 & 56 & 167 & \\
\hline \multicolumn{5}{|l|}{ Age at diagnosis } \\
\hline Mean & 75 & 72 & & \\
\hline Range & $53-87$ & $56-89$ & & \\
\hline Tumour grade & & & & 0.39 \\
\hline 1 & $6(14.3)$ & $1(5.0)$ & 7 & \\
\hline 2 & $27(64.3)$ & $10(50.0)$ & 37 & \\
\hline 3 & $8(19.0)$ & $6(30.0)$ & 14 & \\
\hline NA & $1(2.4)$ & $3(15.0)$ & 4 & \\
\hline Tumour size & & & & 0.71 \\
\hline $\mathrm{T} 1$ & $5(11.9)$ & $4(20.0)$ & 9 & \\
\hline $\mathrm{T} 2$ & $19(45.2)$ & $9(45.0)$ & 28 & \\
\hline T3 & $2(4.8)$ & $2(10.0)$ & 4 & \\
\hline T4 & $11(26.2)$ & $4(20.0)$ & 15 & \\
\hline NA & $5(11.9)$ & $1(5.0)$ & 6 & \\
\hline Nodal status & & & & 0.36 \\
\hline No & $27(64.3)$ & $11(55.0)$ & 38 & \\
\hline N1 & $8(19.0)$ & $7(35.0)$ & 15 & \\
\hline N2 & $1(2.4)$ & $1(5.0)$ & 2 & \\
\hline N3 & $1(2.4)$ & 0 & 1 & \\
\hline$N X$ & $1(2.4)$ & 0 & 1 & \\
\hline NA & $4(9.5)$ & $1(5.0)$ & 5 & \\
\hline Metastasis status & & & & 1.00 \\
\hline MO & $34(80.9)$ & $18(90.0)$ & 56 & \\
\hline M1 & $2(4.8)$ & 0 & 2 & \\
\hline$M X$ & $1(2.4)$ & $1(5.0)$ & 2 & \\
\hline NA & $5(11.9)$ & $1(5.0)$ & 6 & \\
\hline ER score (Allred) & & & & 0.18 \\
\hline 6 & $1(2.4)$ & 0 & 1 & \\
\hline 7 & $6(14.3)$ & $6(30.0)$ & 12 & \\
\hline 8 & 35 (83.3) & $14(70.0)$ & 49 & \\
\hline HER status & & & & 0.69 \\
\hline Negative & $35(83.3)$ & $12(60.0)$ & 47 & \\
\hline Positive & $6(14.3)$ & $3(15.0)$ & 9 & \\
\hline NA & $1(2.4)$ & $5(25.0)$ & 6 & \\
\hline Histological type & & & & 0.73 \\
\hline IDC (no special type) & $18(42.9)$ & $6(30)$ & 24 & \\
\hline ILC & $8(19.0)$ & $4(20)$ & 12 & \\
\hline Mucinous & $1(2.4)$ & 0 & 1 & \\
\hline NA & $15(35.7)$ & $10(50)$ & 25 & \\
\hline Molecular subtype ${ }^{b}$ & & & & 1.00 \\
\hline Luminal A & $21(50.0)$ & $9(45.0)$ & 30 & \\
\hline Luminal B & $20(47.6)$ & $9(45.0)$ & 29 & \\
\hline
\end{tabular}


Table 1 Patient characteristics (Continued)

\begin{tabular}{llll}
\hline & Dormant, $n(\%)$ & Resistant, $n(\%)$ & Total, $n$ \\
\hline HER2 enriched & 0 & $1(5.0)$ & 1 \\
Basal-like & 0 & 0 & 0 \\
Normal-like & 0 & 0 & 0 \\
NA & $1(2.4)$ & $1(5.0)$ & 2 \\
\hline
\end{tabular}

ER oestrogen receptor, IDC invasive ductal carcinoma, ILC invasive lobular carcinoma, NA not available

${ }^{a}$ Fisher exact test ( $p<0.05$, two-tailed)

${ }^{\mathrm{b}}$ At diagnosis by PAM50 (genefu)

\section{Gene expression profiling and analysis}

RNA was extracted from the aqueous phase by column-based purification (RNeasy mini kit, Qiagen) and then labelled and hybridized (HumanHT-12 v4 Illumina BeadChip) according to the manufacturer's protocol (NuGEN) as previously described $[19,20]$. Raw data were detection $(p<0.05, \geq 3$ samples) and quality filtered, $\log _{2}$ transformed, and quantile normalized using the Bioconductor lumi package [21]. Data are publicly available from NCBI GEO under accession GSE111563. The analysis also includes data from 14 patients (42 samples, GSE59515) and 9 patients (24 samples, GSE55374) from previous studies [16, 19] which meet the criteria defined above; the relationship between the samples from these datasets is indicated in Additional file 2 (Table S1). Hierarchical clustering analysis was performed using a complete linkage method and Euclidean distance. Pathway enrichment analysis and visualisation were performed using ReactomePA [22]. Differential gene expression analysis was performed with Rank Products [23]. The significance of differences was evaluated by using unpaired Wilcoxon test for two groups and analysis of variance (ANOVA) with post-hoc Tukey HSD for multiple comparisons.

\section{Proteomics analysis}

Proteins were isolated from the organic phase of Qiazol [24]. Pellets were sonicated and dissolved in 1\% SDS. Proteomics was performed using Thermo $Q$ Exactive plus and Label-free Quantitation (LFQ). Peptides obtained from samples were analysed in mass spectrometry runs; serial samples from the patients were run on the same day. A modified version of Filter Aided Sample Preparation (FASP) was performed using serial digests with lysC and trypsin to generate two orthogonal fractions per sample $[25,26]$. The mass spectrometry spectra generated in each run were used for relative quantitation of individual peptides. Normalization and quantifications of peptides were performed using MaxLFQ and MaxQuant [27]. A total of 6251 protein groups were identified. Data was $\log _{2}$ transformed and missing values were imputed as the minimum observed value in each sample. The data have been deposited to the ProteomeXchange Consortium via the PRIDE [28] partner repository with the dataset identifier PXD009328.

\section{Immunohistochemistry and scoring}

Formalin-fixed paraffin-embedded (FFPE) sections were processed using an automated stainer (Leica Biosystems, Bond III). Heat-induced epitope retrieval for both antibodies was performed by $30-\mathrm{min}$ incubation in citrate-based pH6.0 epitope retrieval (ER1) solution followed by incubation in $3.5 \mathrm{~N} \mathrm{HCl}$ for $15 \mathrm{~min}$ at room temperature as suggested by Haffner et al. [29]. For 5-methylcytosine (5-mC) and 5-hydroxymethylcytosine (5-hmC) detection, mouse monoclonal 5-methylcytosine specific (33D3; Abcam, ab10805) and rabbit polyclonal 5-hydroxylmethylcytosine (Active Motif, 39,769) antibodies were used, respectively. Both antibodies were used at 1/1000 dilution and were incubated for $15 \mathrm{~min}$. Detection was performed using secondary antibody-horseradish peroxidase (HRP) conjugates and substrate-chromogen (3,3'-diaminobenzidine (DAB)). After staining, slides were counterstained with haematoxylin. Nuclear staining in epithelial cells was evaluated using an $\mathrm{H}$-score obtained by multiplying the intensity of the stain (0: no staining; 1 : weak staining; 2 : moderate staining; 3: intense staining) by the percentage of cells (H-score range, 0 to 300$)$.

\section{Results}

Long-term oestrogen deprivation therapy as a model of dormancy and acquired resistance

A cohort of 62 primary breast cancer patients receiving at least 4 months of oestrogen deprivation therapy (Fig. 1a) and initially responding were stratified into two groups, 'dormant' and 'acquired resistant' based on dynamic changes in tumour size and proliferation (see methods and Fig. 1b). Patient-matched sequential samples were available at three time points: before ( $\leq 0$ days), early (13-120 days), and long-term (> 120 days) treatment. Dormant and acquired resistant samples were distributed uniformly with respect to time on treatment, and duration at each time point was not significantly different between response groups (Table 1). For long-term treatment, the mean and range were 186 (121-884) days 


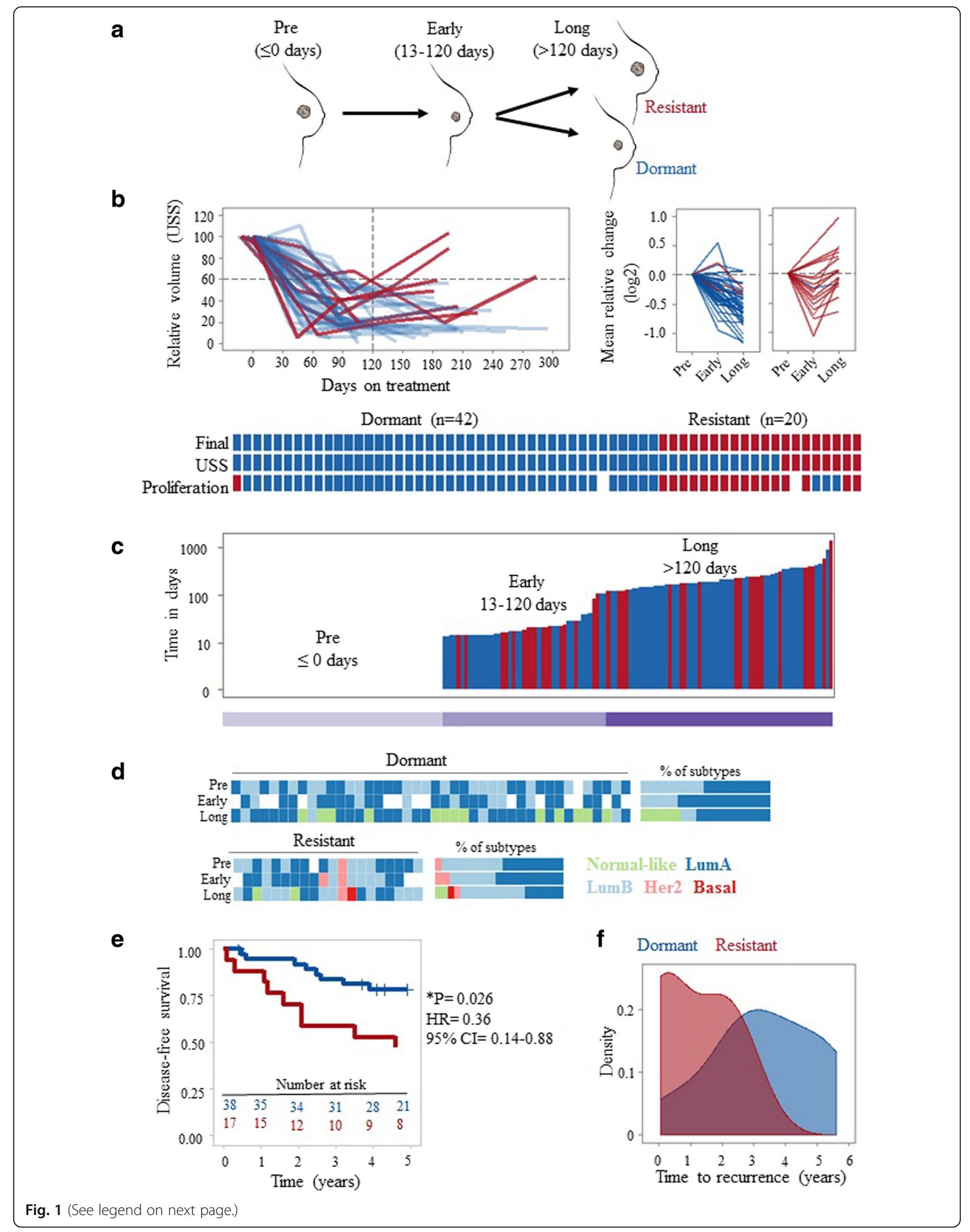


(See figure on previous page.)

Fig. 1 Long-term oestrogen deprivation therapy as a clinical model to investigate breast cancer dormancy and acquired resistance. a Extended (4-45 months) letrozole treatment was exploited as a clinical model of breast cancer dormancy and acquired resistance. Sequential clinical samples from the same patient with no surgery and extended treatment were used to model clinical breast cancer dormancy and resistance. Before (pre, $\leq 0$ days), early-on (early, 13-120 days) and long-term (long, > 120 days) neoadjuvant aromatase inhibitor therapy with letrozole. b Dynamic change in tumour size by ultrasound scan (USS) and mean expression of proliferation markers MKI67, PCNA, and MCM2 were used to classify patients into two categories: dormant (blue) and resistant (red). Overall comparisons of classifications per patient based on USS and mean change in proliferation markers with final classification are shown. c The duration of letrozole treatment (days) for samples from dormant (blue) and resistant (red) patients. Each bar represents a sample. Samples are ordered by time on treatment. $\mathbf{d}$ Intrinsic subtype classification by PAM50 of samples at each time point. Stacked bar graphs on the right show the percentage of each subtype of samples from dormant and resistant patients. e Kaplan-Meier plot showing disease-free survival probability in patients with dormant versus resistant tumours (log-rank test). Disease-free sunvival was defined from time of surgery. $\mathbf{f}$ Density plot showing the distribution of time to recurrence (in years; defined from time of surgery) in patients with dormant and resistant tumours. Cl confidence interval, HR hazard ratio, LumA luminal A, LumB luminal B

and 226 (121-1366) days for patients with dormant and acquired resistant tumours, respectively (Fig. 1c).

There were no significant differences in patient clinico-pathological features between response classes before treatment (Table 1). However, prediction analysis of microarray (PAM)50 intrinsic molecular subtypes were found to change during oestrogen deprivation therapy (Fig. 1d). These changes were consistent with known associations with outcome, with all dormant tumours either remaining the same or switching to better prognosis luminal $\mathrm{A}$ or normal-like tumours. For resistant tumours, however, $25 \%$ (5 out of 20) switched to a subtype of worse prognosis (Fig. 1d). The proportion of luminal B tumours characterised by reduced endocrine sensitivity and higher proliferation was higher in resistant tumours compared with dormant tumours under early (35\% versus $27 \%$ ) and long-term (50\% versus $12 \%$ ) treatment (Fig. 1d; stacked bar graphs on the right). The PAM50 defines breast cancer into four intrinsic molecular subtypes: luminal A, luminal B, HER2enriched, and basal-like [30]. PAM50 intrinsic subtyping has been shown to provide additional prognostic value to standard clinicopathological factors where luminal A tumours had a significantly better outcome than luminal B, HER2-enriched, and basal-like tumours [31].

As expected, Kaplan-Meier survival analysis demonstrated significantly worse outcomes for patients with resistant tumours compared with patients with dormant tumours ( $\log$ rank, $p=0.026$; Fig. 1e). Recurrence rates for patients with dormant and resistant tumours were $21 \%(9 / 42)$ and $45 \%$ (9/20), respectively. Moreover, patients with resistant tumours suffered significantly earlier recurrences compared with patients with dormant tumours $(p=0.05$; range 26947 versus 136-2042 days; Fig. 1f). Disease-free survival and time to recurrence were defined from time of surgery, not from the time of diagnosis, since patient classification was performed based on change in tumour size by USS and proliferation by gene expression at on-treatment and surgery time points.

\section{Distinct transcriptomic changes under long-term letrozole treatment}

Unsupervised analysis was performed to consider whether sequential samples displayed greater similarity between response classes or treatment duration. Hierarchical clustering using the 500 genes with the highest variance across all samples revealed two main subclasses, seemingly driven by time on treatment, with resistant and dormant tumours indistinguishable (Fig. 2a). The dominant pattern was that the samples of the same patient usually clustered together (Fig. 2a).

When long-term treated samples were considered alone, two clusters did emerge, the larger of which contained mostly dormant samples (79\%), whilst the second had a roughly even proportion of dormant (48\%) and resistant (52\%) samples (Fig. 2a). Similarly, a multidimensional scaling (MDS) plot for the 500 genes with the highest variance across all time points revealed consistent changes over time in response to treatment for both dormant and acquired resistant samples (Fig. 2b), although long-term dormant samples were much more distinguishable from pre-treatment samples than the long-term acquired resistant samples (Fig. 2b).

Correlations between tumours from different individuals (inter-patient) remained similar at each time point and were not different between response classes (data not shown) as corroborated by hierarchical clustering analysis with all samples and across all the time points. However, correlations of the transcriptome between matched sequential samples (intra-patient) revealed that pre-treatment samples were significantly $(p=0.01)$ less similar to their long-term treated pairs (median 0.89, range 0.74-0.95) than their early treatment pairs (median 0.91, range 0.84-0.95) (Fig. 2c). However, when divided by dormancy status this finding was only significant $(p=0.01)$ for dormant tumours (Fig. 2c), suggesting that dormant tumours continue to diverge transcriptionally whereas acquired resistant tumours do not consistently differ after initial or extended treatment, as mirrored in the MDS representation (Fig. 2b).

Perhaps surprisingly, oestrogen, progesterone, and androgen receptors and their target genes [32] were not 


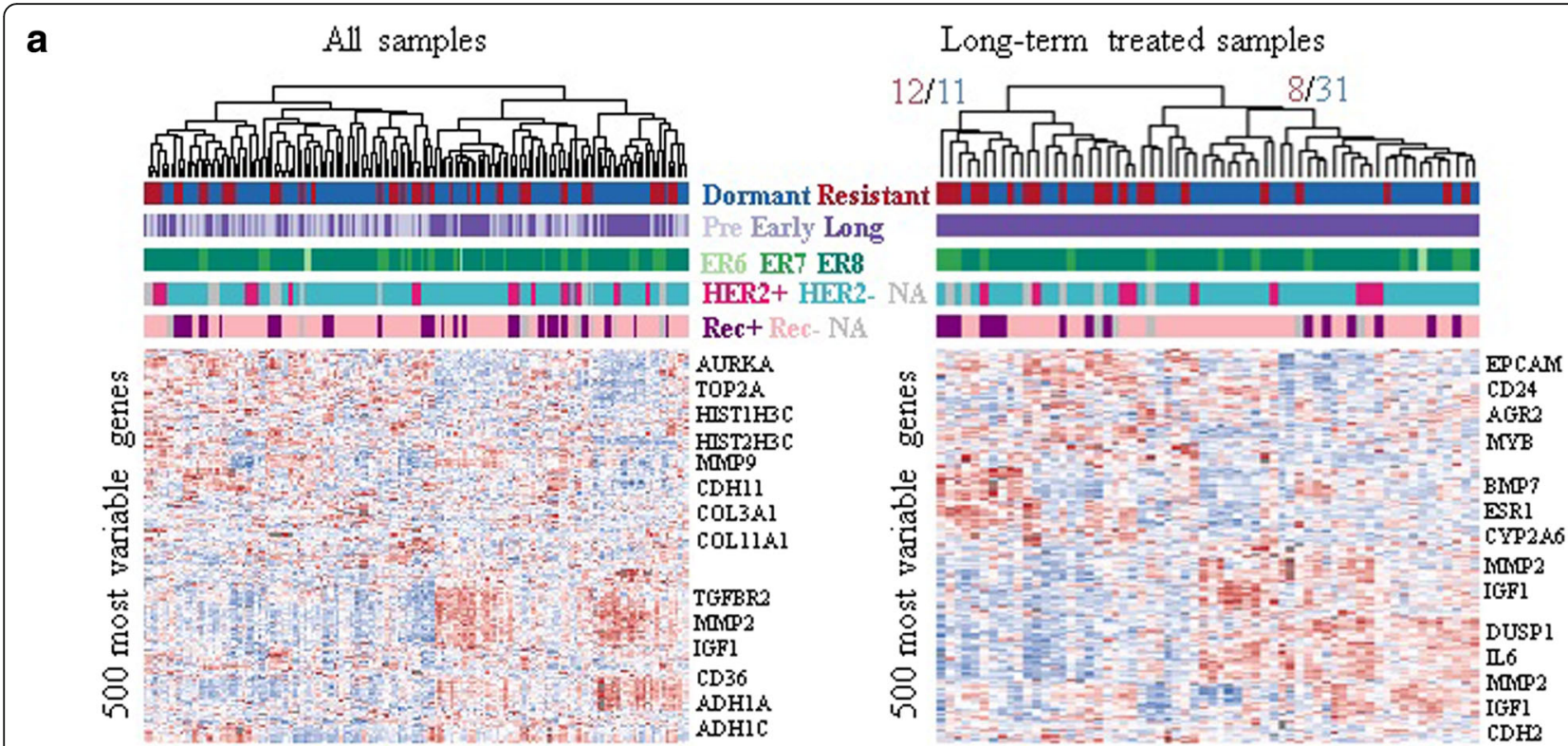

b

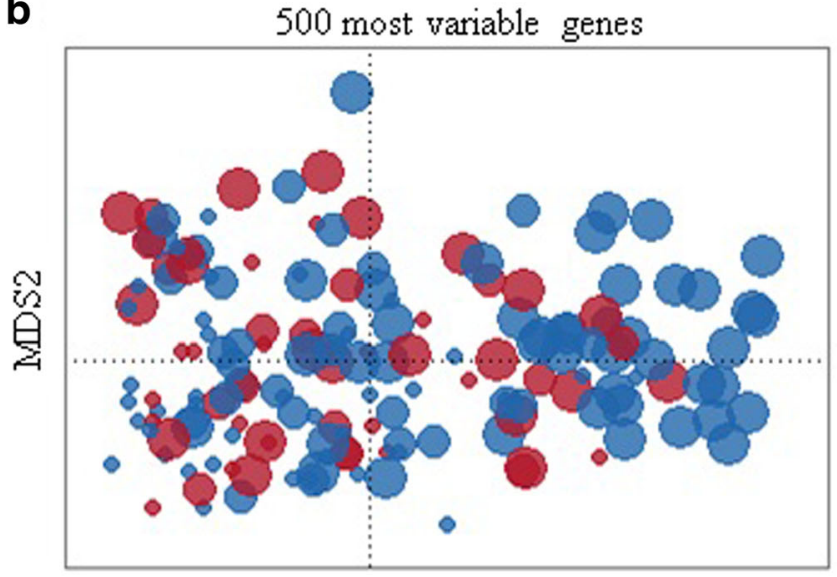

C

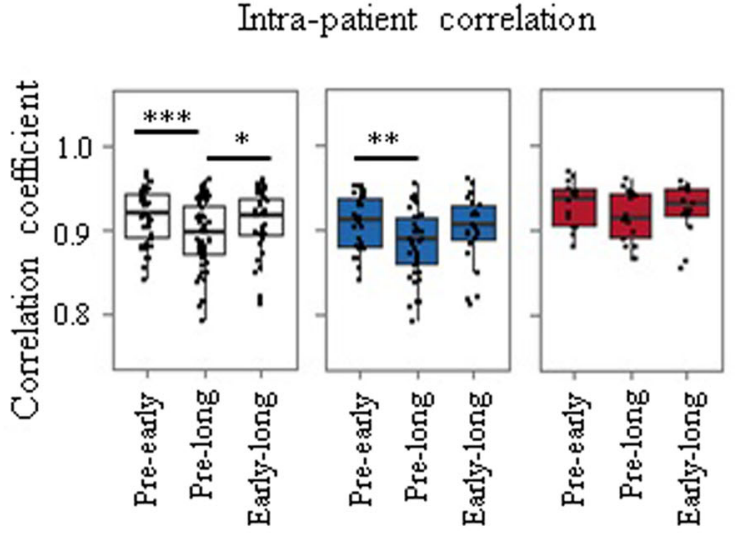

Fig. 2 Distinct transcriptomic changes during long-term aromatase inhibitor treatment. a Unsupervised hierarchical clustering with most variant 500 genes across all samples and long-term treated samples. ER6, ER7, ER8 correspond to oestrogen receptor (ER) Allred scores 6, 7, and 8, respectively. $\mathbf{b}$ Multidimensional scaling (MDS) plot using the 500 genes with the highest variance across all time points. Each dot corresponds to a sample and sizes represent the duration of treatment. $\mathbf{c}$ Intra-patient (comparison of samples from the same patient) correlations of transcriptome are shown. Dormant (blue); resistant (red); before (pre, $\leq 0$ days), early-on (early, 13-120 days), and long-term (long, > 120 days) neoadjuvant aromatase inhibitor therapy with letrozole. ${ }^{* *} p<0.001 ;{ }^{* *} p<0.01 ;{ }^{*} p<0.05$. NA not available, Rec + recurrence, Rec- recurrence free

differentially expressed between long-term treated dormant and resistant tumours (data not shown).

\section{Changes in gene expression/pathways following long- term letrozole treatment}

To consider whether the gene expression changes due to treatment in the dormant and acquired resistant tumours were the same or distinct we initially considered them separately. Pairwise rank product analysis (pre- versus long-term treatment, false discovery rate $(\mathrm{FDR})<0.01$ ) identified 2319 genes significantly differentially expressed (1063 downregulated and 1256 upregulated) between long-term treated and pre-treatment dormant tumours (Additional file 2: Table S2). These genes were significantly enriched $(p<0.01)$ for a total of 62 and 26 pathways, respectively (Additional file 2: Table S3), including reductions in cell cycle, senescence, DNA methylation, and an increase in extracellular matrix (ECM) organization. These findings are consistent with previous studies of patient-matched sequential samples treated with 
oestrogen deprivation therapy [16-18]. Acquired resistant tumours displayed much fewer consistently differentially expressed genes $(238$; 63 downregulated and 175 upregulated) between long-term treated and pre-treatment samples (Additional file 2: Table S4). Genes that were upregulated in resistant tumours (pre-treatment versus long-term treatment) were enriched for several of the same pathways as dormant tumours (ECM organization, elastic fibre formation, and platelet degranulation), but downregulated genes were much more variable (Additional file 2: Table S5; Fig. 3a, b).

Having determined that dormant and acquired resistant tumours have somewhat distinct changes during treatment at the molecular level, the question remained as to whether these changes tend to occur at earlier time points or were specific to long-term treatment. For dormant tumours, differential expression begins early on, but becomes more pronounced at later time points (Fig. 3a). Downregulated genes (pre-treatment versus long-term treatment) were most evident at early-on treatment for resistant tumours, consistent with their initial response to treatment, whilst upregulated genes (pre-treatment versus long-term treatment) were most changed after long-term treatment, potentially suggesting that these genes may mediate acquired resistance (Fig. 3b). We further examined whether differentially expressed genes between pre-treatment versus long-term treatment identified in each response class (dormant and resistant) were shared (Fig. 3c, d). Both downregulated and upregulated genes identified in resistant tumours were significantly changed $(p<0.01)$ in dormant tumours (Fig. 3d). However, only upregulated genes identified in dormant tumours were significantly upregulated in resistant tumours without any change in downregulated genes (Fig. 3c), implicating a partial lack of response to treatment at the molecular level in acquired resistance patients.

\section{A potential role of epigenetic regulation in acquired resistance}

The above findings suggest that therapy-induced dynamic changes in gene expression and pathways are common features of long-term treatment, rather than being specific to dormant or resistant phenotypes. This led us to perform comparative analysis of dormant and acquired resistant tumours at the long-term time point to identify any specific differences. Unpaired rank product analysis (FDR $<0.01)$ revealed a total of 419 genes (170 downregulated and 249 upregulated) to be differentially expressed between long-term treated dormant and resistant tumours (Additional file 2: Table S6; Fig. 4a). These genes were significantly enriched in 27 pathways $(p<0.05)$, including several epigenetics-related pathways, including "DNA methylation", "PRC2 methylates histones and DNA", "histone acetyl transferases (HATs) acetylate histones", and "epigenetic regulation of gene expression", as well as senescence and cell cycle (Additional file 2: Table S7; Fig. 4b). Examination of the expression of these genes alone demonstrated that they could partially separate dormant from the majority of resistant tumours (Fig. 4c). Single-sample gene set enrichment analysis (ssGSEA) [33] was performed to quantitatively score the activity of differentially expressed genes in every sample. The score of differentially upregulated genes between long-term treated dormant and resistant tumours was significantly higher in acquired resistant compared with dormant tumours under early treatment $(p<0.05)$ as well as long-term treatment $(p<0.001)$ (Fig. $4 \mathrm{~d})$.

Our results prompted us to examine whether the changes we observed in clinical samples were similarly changed in experimental models of resistant breast cancer cells. Oestrogen receptor-positive MCF7 cells stably transfected with the aromatase gene (MCF7aro cells) and long-term oestrogen-deprived (LTED) breast cancer cells have been widely used to understand mechanisms of aromatase inhibitor resistance in vitro. Examining two publicly available gene expression datasets (GSE10879 and GSE10911) demonstrated that genes differentially expressed (upregulated) between acquired resistant and dormant tumours (a total of 249) were significantly enriched in aromatase inhibitor-resistant cells compared with sensitive/control cells (Fig. 5a). A total of 211 and 174 out of 249 genes were present in GSE10879 and GSE10911, respectively. In two out of three in-vitro studies with dynamic gene expression data from LTED MCF7 cells, an initial decrease in ssGSEA scores mimicking the dormancy/responsive state was followed by a later increase representing acquired resistance (Fig. 5b), further validating our results and emphasizing the utility of these in-vitro models. Interestingly, no significant difference was observed in tamoxifen- and fulvestrant-resistant MCF7 cells compared with drug-sensitive control cells (Fig. 5c) suggesting the specificity of the results to resistance to aromatase inhibitor therapy.

In addition, proteomic analysis of a subset of samples was performed which revealed differential expression in 656 proteins (279 downregulated, 377 upregulated) between long-term treated dormant and resistant tumours (rank product; $p<0.05 ; n=10$; Additional file 2: Table S8; Fig. 5d). A total of 36 features including S100P and HIST2H3A (H3.2) overlapped between proteomics and transcriptomics, validating the results with a different approach.

Furthermore, differentially expressed genes were uploaded to Enricher (ENCODE Histone modification 2015 dataset) [34] to determine histone modification enrichment. Two H3 lysine methylation modifications (H3K27me3 and H3K4me1) were enriched 


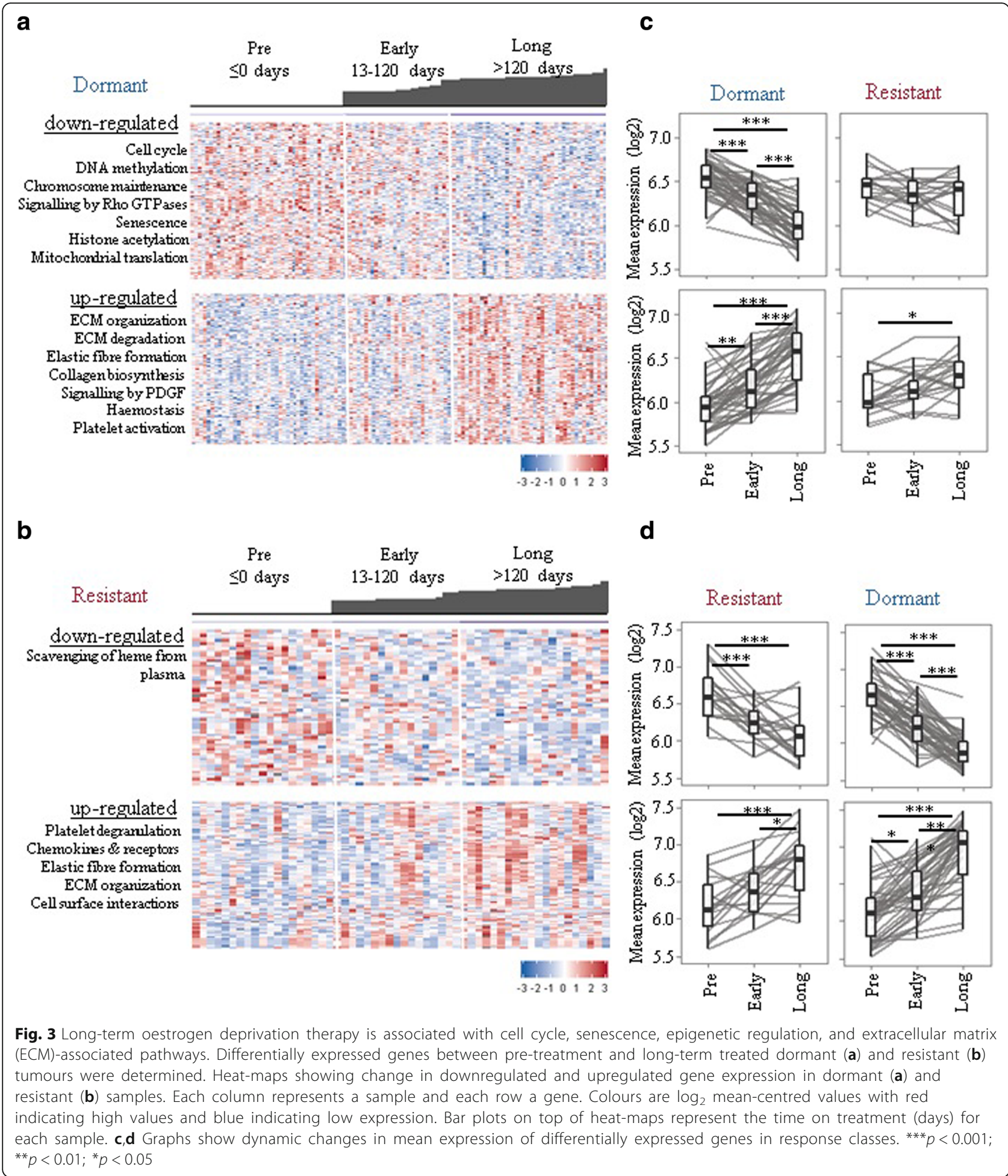

significantly (adjusted $p=0.0003$ and $p=0.004$, respectively) whereas no enrichment for histone acetylation was determined.

To further validate the gene expression results, immunohistochemical evaluation of FFPE sections revealed significantly lower global $5-\mathrm{mC}$ and $5-\mathrm{hmC}$ levels in resistant tumours compared with dormant tumours under extended treatment (Fig. 6a, b). Significantly lower 5 -hmC levels in acquired resistant compared with dormant tumours were also observed at early treatment (Fig. 6b), suggesting hypomethylation may be predictive of emergence from dormancy. 


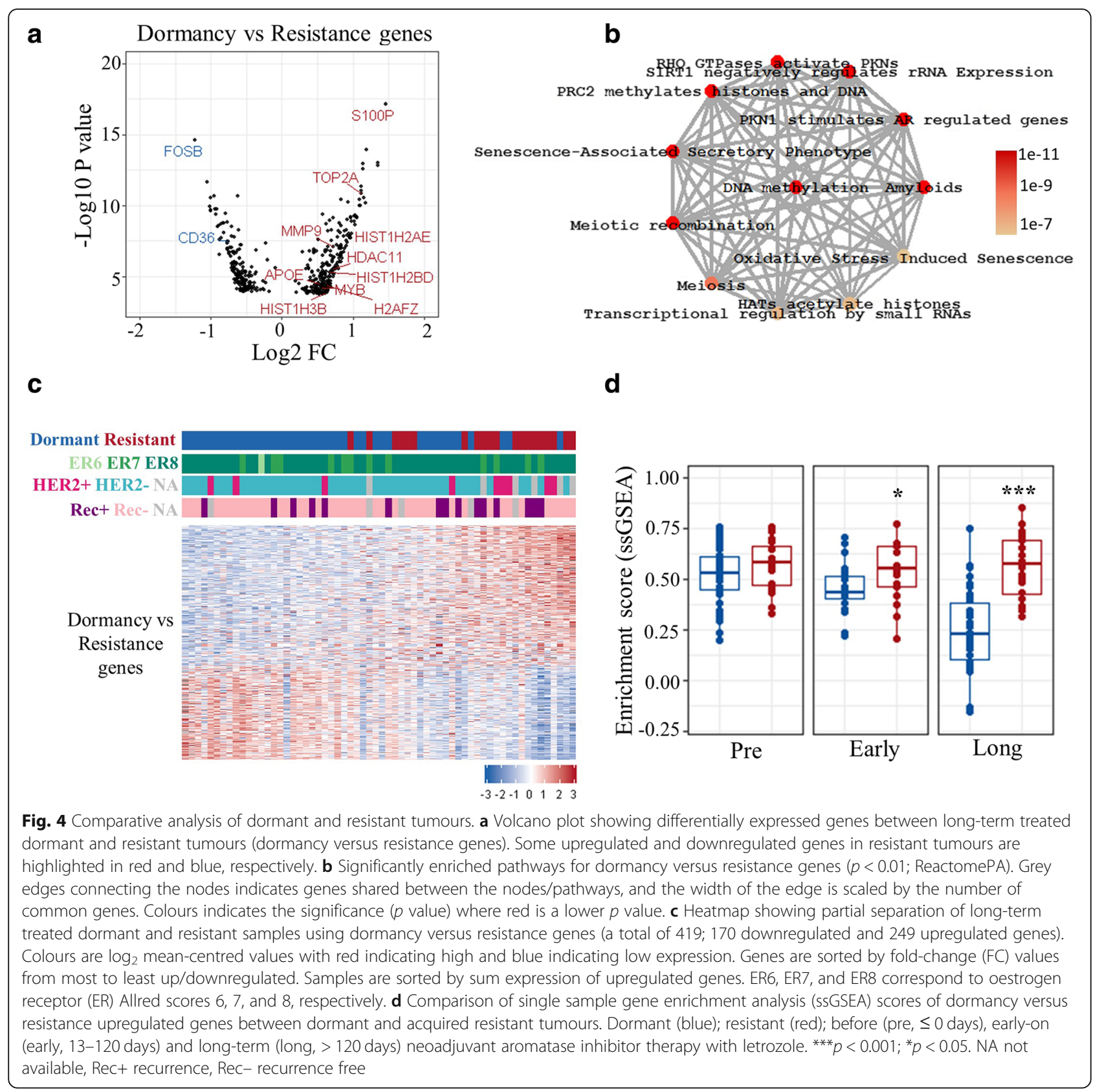

\section{Discussion}

Understanding the mechanisms underlying the maintenance of and escape from dormancy have great importance considering that most cancer-related deaths are caused by metastasis rather than the primary tumour. In this study, we describe the first sequential patient-matched clinical dataset of extended oestrogen deprivation therapy in breast cancer. The results highlight the difficulty of distinguishing dormant and resistant tumours, with dynamic molecular changes of treatment being highly similar between the groups. However, comparative analysis revealed a set of genes significantly upregulated in resistant tumours compared with dormant tumours within the first months of letrozole treatment suggesting a predictive role for changes in DNA methylation.

Failure to reduce proliferation after 2 weeks of oestrogen deprivation therapy $[16,35]$ may well identify patients that are innately resistant; however, acquired resistance remains a greater challenge in terms of identifying biomarkers and appropriate alternative or combination therapies [36]. Many of the transcriptomic changes identified in long-term treated dormant tumours are shared by some, but not all, resistant 


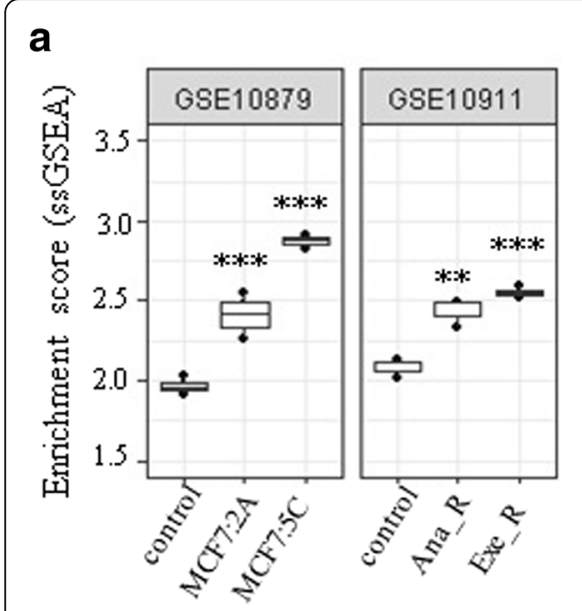

b

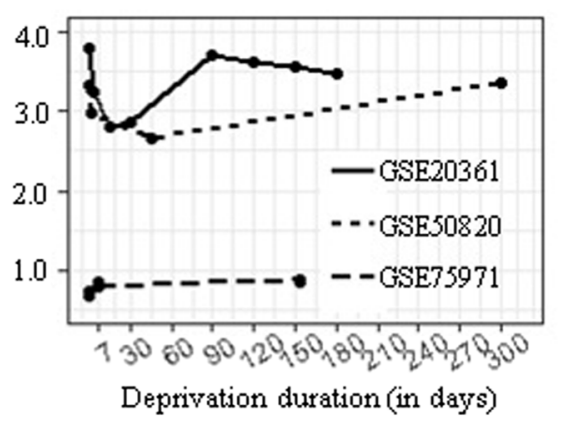

C

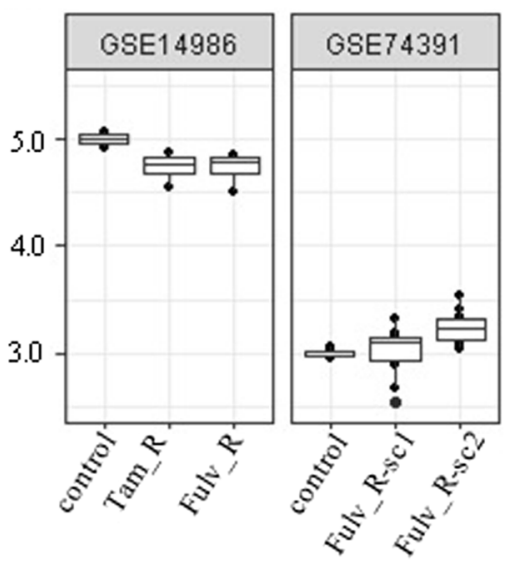

d

Proteomics analysis

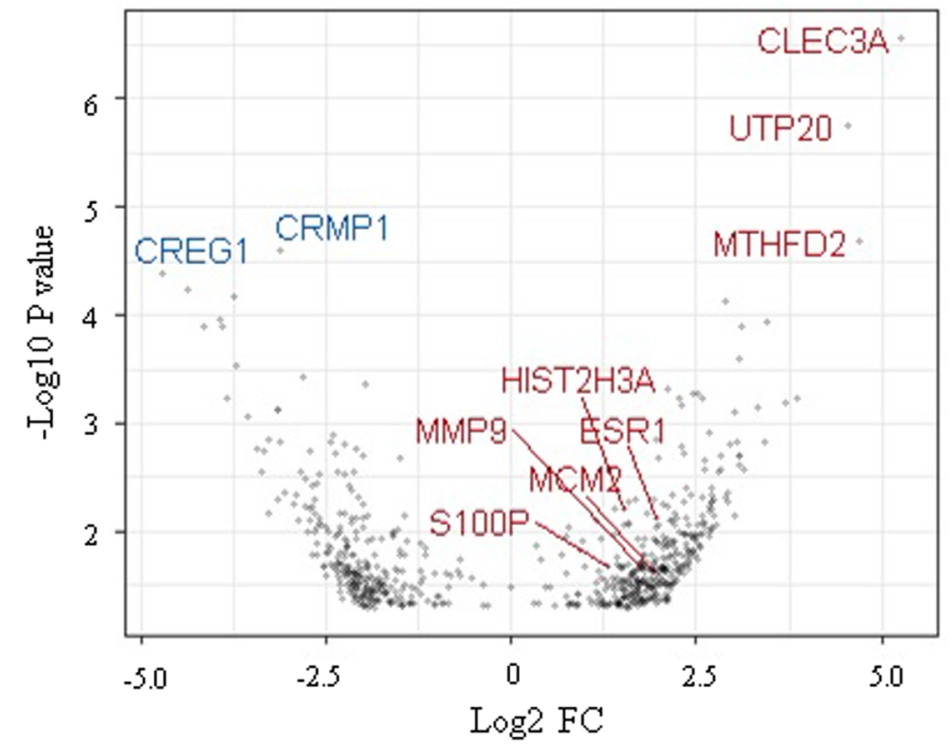

Fig. 5 Validation of results using in-vitro gene expression data from resistant cell lines and proteomics analysis. a Normalised enrichment scores of differently upregulated genes (a total of 249) between long-term treated dormant and resistant tumours calculated using single sample gene set enrichment analysis (sSGSEA) in aromatase inhibitor-resistant cells. Scores were significantly higher $\left({ }^{* *} p<0.01,{ }^{* * *} p<0.001\right)$ in two aromatase inhibitor-resistant cell lines, MCF7:2A and MCF7:5C, which were clonally derived from MCF7 breast cancer cells following long-term oestrogen deprivation (LTED) compared with control/sensitive MCF7 cells $(n=4)$. Anastrozole-resistant (Ana_R) and exemestane-resistant (Exe_R) MCF7aro cells had significantly higher scores compared with control $(n=3)$. b Dynamic changes in enrichment scores of LTED MCF7 cells in three different datasets. c Scores in tamoxifen-resistant (Tam_R) and fulvestrant-resistant (Fulv_R) and drug-sensitive (control) MCF7 cells $(n=4, n=10)$. d Volcano plot showing differentially expressed proteins between long-term treated dormant and resistant tumours $(p<0.05)$. Some overlapping features between transcriptomics and proteomics analysis and the most upregulated and downregulated proteins are highlighted in red and blue, respectively. FC fold-change, sc subclone

tumours, providing further evidence of resistance heterogeneity [37] where dormant tumours share similar molecular changes, but there are likely to be a variety of escape mechanisms that lead to acquired resistance.

In the present study, paired differential expression analysis demonstrated that dormant tumours continue to change under long-term treatment. Some of the identified dormancy-related pathways such as cell cycle arrest and senescence have established roles in metastasis dormancy [38], further supporting the relevance of our clinical model, with the senescence-associated secretory phenotype (SASP) recently suggested to regulate breast cancer dormancy and relapse [39]. As in short-term responsive tumours [16], ECM organization and degradation were significantly upregulated in dormant tumours. ECM remodelling and its degradation by 


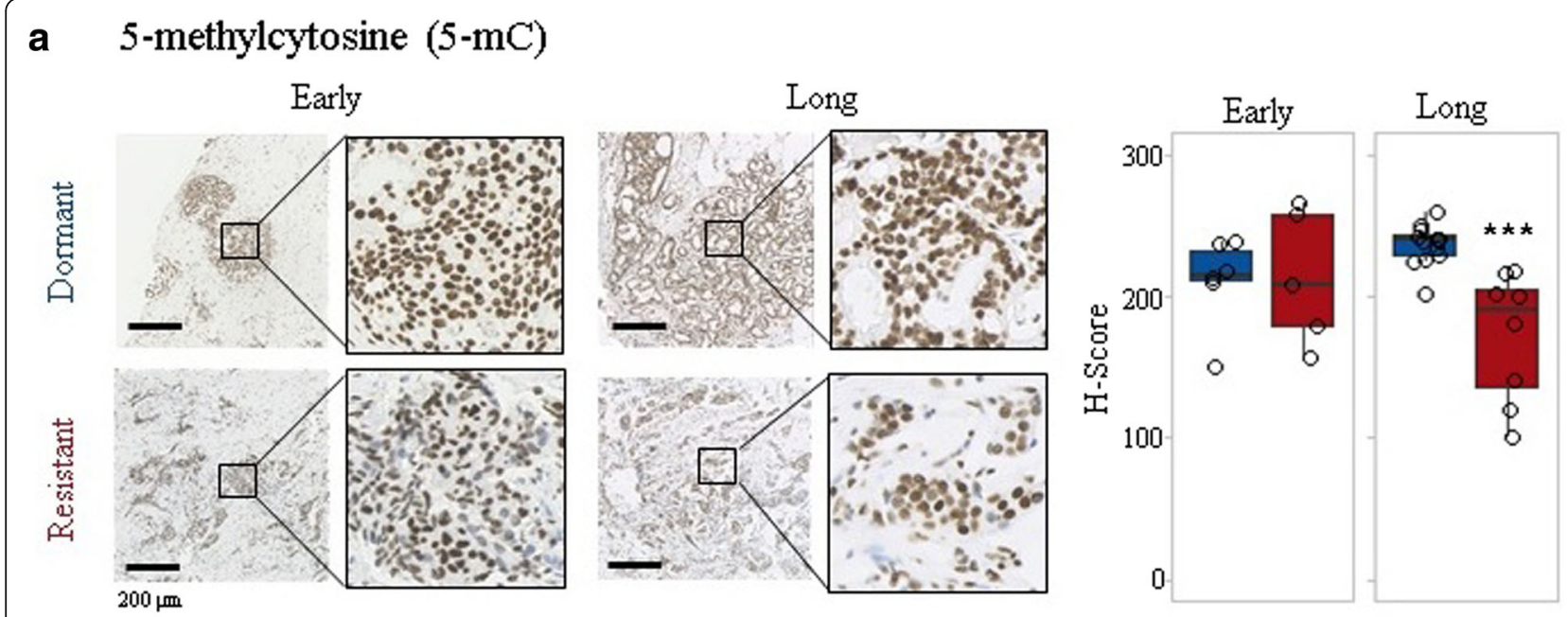

\section{b 5-hydroxymethylcytosine $(5-\mathrm{hmC})$}
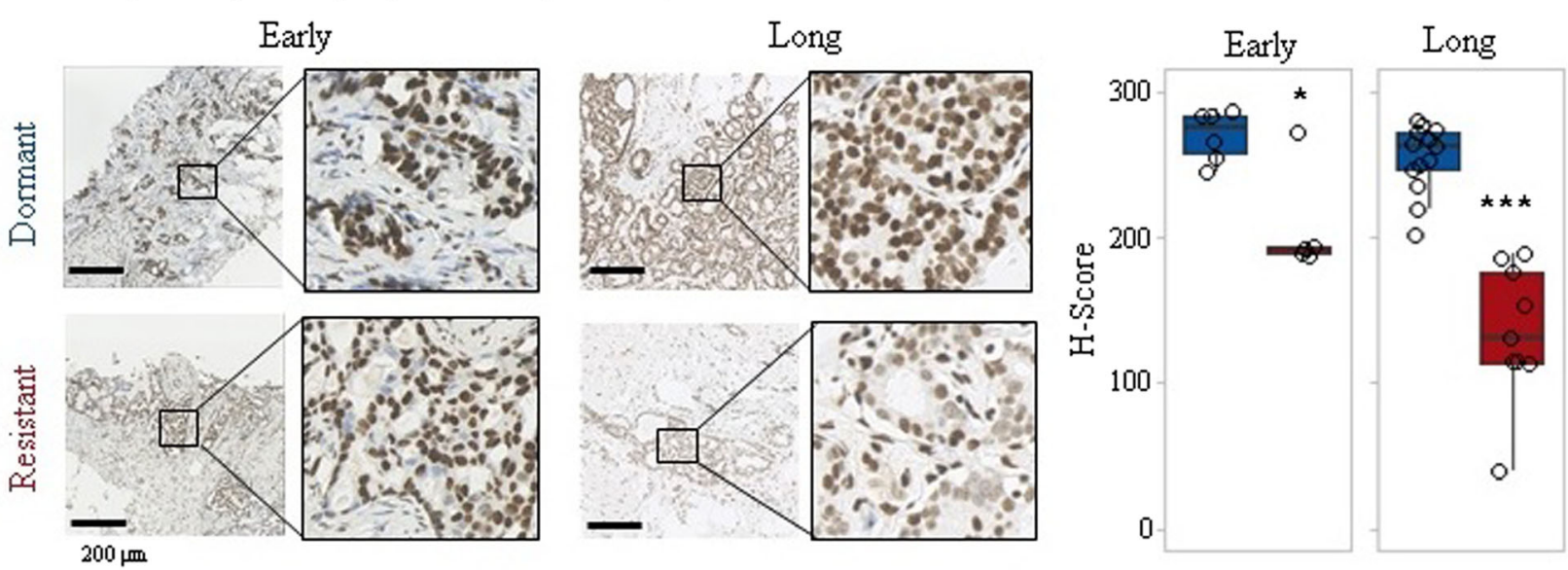

Fig. 6 Immunohistochemical evaluation of methylation markers. a 5-methylcytosine (5-mC) and b 5-hydroxymethylcytosine (5-hmC) levels were determined in FFPE sections from letrozole-treated samples. Representative images in dormant and resistant tumours are shown. Boxplots show distributions of semi-quantitative intensity scores of 5 - $\mathrm{mC}\left({ }^{*} p<0.05\right.$, $\left.{ }^{* *} p<0.001 ; n=5-12\right)$ and 5 -hmC (*** $\left.p<0.001 ; n=5-13\right)$ levels in dormant and resistant tumours. Early-on (early, 13-120 days) and long-term (long, > 120 days) neoadjuvant aromatase inhibitor therapy with letrozole

matrix metalloproteases (MMP) have previously been suggested to regulate the switch between dormancy and metastatic growth [40]. Despite histological confirmation that each tumour sample contained at least $60 \%$ tumour, we acknowledge that the results presented are of intact whole tissue and potentially limited by minor variations in tumour cellularity or the proportion of stoma which could affect gene expression.

The most transcriptionally upregulated gene in resistant tumours S100P, previously shown to be an inducer of breast cancer metastasis correlated with decreased survival [41]. S100P, a small calcium-binding protein mediating $\mathrm{Ca}^{2+}$-dependent signalling pathways, has distinct functions in normal tissue and cancer, including human embryonic development and breast cancer initiation [42]. Recently, S100P hypomethylation in blood was demonstrated to be inversely correlated with tissue S100P expression and significantly associated with breast cancer, implicating $S 100 P$ as a potential diagnostic marker [43]. High plasma S100P levels have also been correlated with poor prognosis in metastatic breast cancer patients, with levels decreasing following treatment, suggesting a role of S100P in dynamic monitoring of response [44]. In the present study, S100P gene expression and protein levels were significantly higher in resistant tumours after long-term treatment, as well as being differentially expressed before treatment, supporting its potential role as a therapeutic target [45] and a predictive marker.

Comparative analysis of dormant and resistant samples after extended treatment revealed enrichment for a set of genes with a role in DNA methylation and histone 
acetylation/deacetylation. Epigenetic alterations are recognized to occur in breast cancer. DNA methyltransferase (DNMT) and histone deacetylase (HDAC) inhibitors have been shown to exert encouraging effects on the disease [46]. Recently, the potential role of epigenetic changes in regulating dormancy and reactivation state has been suggested to explain the reversible (on/off) nature of dormancy [47].

Breast cancer "CpG island methylator phenotype" (CIMP), as revealed by genome-wide methylation analysis of metastatic breast cancers where a large number of genes are hypermethylated, has been suggested to be informative for metastatic potential [48]. A significant correlation between pre-treatment global DNA methylation with neoadjuvant chemotherapy response in rectal cancer has been reported [49]. Although DNA hypomethylation was the first epigenetic alteration identified in cancer, its molecular process and effects are not yet well understood [50]. In addition, 5-hmC levels were shown to correlate with differentiation status, with higher levels when more differentiated [29]. In addition, alterations in DNA methylation in LTED MCF7 cells have been previously reported [51]. Our results provide evidence for the loss of a global DNA methylation process in resistant tumours and strengthen the case to use these models for further study. The global decrease in 5-mC may account for the observed reduction in 5 -hmC levels since $5-\mathrm{mC}$ is converted to $5-\mathrm{hmC}$. On the other hand, at the early-on time point, 5-hmC levels were significantly reduced with no significant change in 5-mC levels, suggesting an independent role of the 5-hmC mark. Hypomethylated cancer cells have been suggested to be selected to form tumours with increased malignancy [50]. We suggest that hypomethylation in resistant tumours may reflect a de-differentiation process inducing stem cell-like cell formation. Determining the time point at which that hypomethylation starts, which would allow intervention before it starts to prevent resistance to therapy, needs further investigation.

The main genes significantly enriched for epigenetics-associated pathways in the present study are core histone (H3, H4, H2B) genes. Well-known epigenetics-associated genes such as DNMT were not differentially expressed in the present study. Therefore, it might be suggested that observed changes in histone gene levels may simply reflect the high proliferation rate in resistant tumours since transcription of these histone genes are replication-dependent and their mRNA levels increase during DNA replication [52]. However, deregulation of histone $\mathrm{H} 2 \mathrm{~A}$ and $\mathrm{H} 2 \mathrm{~B}$ was associated with anthracycline resistance in breast cancer cells and reversed by HDAC small molecule inhibitors [53]. Furthermore, upregulation of replication-dependent core histone proteins has been suggested to be a selective indicator of ER-mediated MCF7 cell proliferation regardless of the proliferation rate [54]. Also, observed global loss of DNA methylation in resistant tumours suggests dynamic regulation of gene transcription under letrozole therapy. Therefore, histone upregulation and alterations in epigenetic pathways observed in our study may play a role in resistance to endocrine deprivation therapy, rather than simply mirroring the degree of proliferation.

Our results indicate alterations both in DNA methylation and histone modifications. HDAC inhibitors, which have been shown to regulate DNA methylation [55], may be successful clinically as second-line drugs alone or in combination following oestrogen deprivation therapy failure as there is growing evidence for their tumour selective action [56, 57]. A time-dependent role for HDACs in leukaemia has been shown [58] and may also be critical in determining when to start HDAC inhibition therapy to successfully treat tumours resistant to oestrogen deprivation therapy. Whether or not the epigenetic alterations are triggers of re-awakening and if the timely use of epigenetic drugs can prevent acquired resistance warrants further investigation.

\section{Conclusions}

We have performed the first study of sequential tumour samples from breast cancer patients receiving extended neoadjuvant oestrogen deprivation therapy as a clinical model of dormancy and acquired resistance. Our analysis suggests that molecular differences between dormant and resistant tumours are initially subtle, becoming more obvious only after extended treatment. This study emphasizes that alterations in DNA methylation in the first months of treatment may predict which patients will eventually develop acquired resistance.

\section{Additional files}

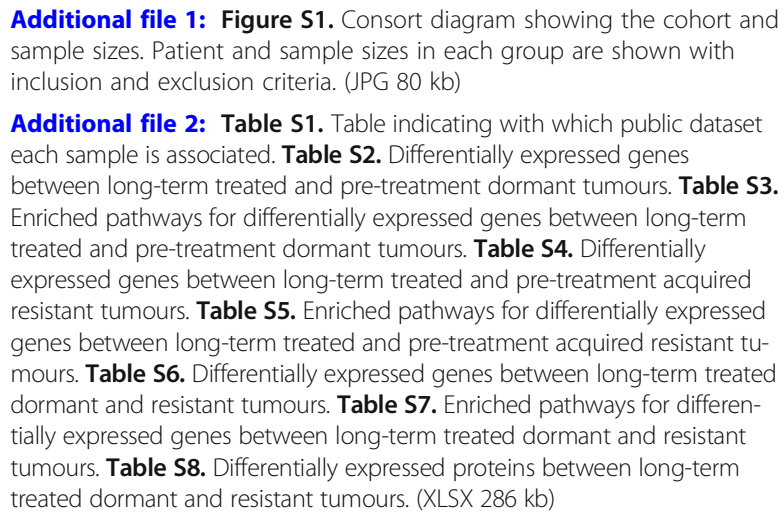

Additional file 2: Table S1. Table indicating with which public dataset each sample is associated. Table S2. Differentially expressed genes between long-term treated and pre-treatment dormant tumours. Table S3. Enriched pathways for differentially expressed genes between long-term treated and pre-treatment dormant tumours. Table S4. Differentially expressed genes between long-term treated and pre-treatment acquired resistant tumours. Table S5. Enriched pathways for differentially expressed genes between long-term treated and pre-treatment acquired resistant tumours. Table S6. Differentially expressed genes between long-term treated dormant and resistant tumours. Table S7. Enriched pathways for differentially expressed genes between long-term treated dormant and resistant tumours. Table S8. Differentially expressed proteins between long-term treated dormant and resistant tumours. (XLSX $286 \mathrm{~kb}$ )

\section{Abbreviations}

5-hmC: 5-Hydroxymethylcytosine; 5-mC: 5-Methylcytosine; CIMP: CpG island methylator phenotype; DAB: 3,3'-Diaminobenzidine; DNMT: DNA 
methyltransferase; ECM: Extracellular matrix; ER: Oestrogen receptor; FASP: Filter Aided Sample Preparation; FDR: False discovery rate; FFPE: Formalin-fixed paraffin-embedded; HAT: Histone acetyl transferase; HDAC: Histone deacetylase; HRP: Horseradish peroxidase; LFQ: Label-free Quantitation; LTED: Long-term oestrogen deprived; MDS: Multidimensional scaling; MMP: Matrix metalloproteases; SASP: Senescence-associated secretory phenotype; ssGSEA: Single-sample gene set enrichment analysis

\section{Acknowledgements}

We are grateful for the help and support of the Genetics Team at the Edinburgh Wellcome Trust Clinical Research Facility under the direction of Lee Murphy.

\section{Funding}

The work was supported Marie Skłodowska-Curie Individual Fellowship (H2020-MSCA-IF, 658170) to CS. AHS and JMD are very grateful for funding provided by Breast Cancer Now. The work was partly supported by Welcome Trust Institutional Fund (ISSF3) to CS and AHS

\section{Availability of data and materials}

The microarray dataset generated during the current study is available in NCBI GEO (www.ncbi.nlm.nih.gov/geo) under accession GSE111563. The analysis also includes previously published microarray data under accession numbers GSE59515 and GSE55374. The proteomics dataset generated during the current study is available in PRIDE (www.ebi.ac.uk/pride/archive) with the identifier PXD009328. Publicly available resistant cell line gene expression datasets GSE10879, GSE10911 [59], GSE20361 [60], GSE50820 [61], GSE75971 [62], GSE14986 [63], GSE74391 [64] were also analysed.

\section{Authors' contributions}

AKT, JMD, and AHS conceived the study. CS and AKT generated the transcriptome dataset. JW conducted proteomics and supported proteomics data analysis. DAP, AL, and AHS provided help with the data analysis. AF and LR provided technical support with tissue collection and processing. LR, JST, and JMD co-ordinated the collection and assessment of clinical samples. CS analysed and interpreted the data and drafted the manuscript. AHS supervised the project and helped to write and edit the manuscript. All authors read and approved the final manuscript.

\section{Authors' information}

Not applicable.

\section{Ethics approval and consent to participate}

All patients provided informed consent and sample collection was approved by the local research ethics committee (Lothian Local Research Ethics Committee 03, REC Reference number 07/S1103/26, approval date 13 August 2007).

\section{Consent for publication}

Not applicable.

\section{Competing interests}

The authors declare that they have no competing interests.

\section{Publisher's Note}

Springer Nature remains neutral with regard to jurisdictional claims in published maps and institutional affiliations.

\section{Author details}

${ }^{1}$ Applied Bioinformatics of Cancer, University of Edinburgh Cancer Research UK Centre, MRC Institute of Genetics and Molecular Medicine, Edinburgh, UK. 2Department of Pharmacology, Faculty of Pharmacy, Ege University, 35040 Izmir, Turkey. ${ }^{3}$ Edinburgh Breast Unit, Western General Hospital, Edinburgh, UK. ${ }^{4}$ Mass Spectrometry Unit, MRC Institute of Genetics and Molecular Medicine, Edinburgh, UK
Received: 10 July 2018 Accepted: 19 December 2018

Published online: 07 January 2019

\section{References}

1. EBCTCG. Aromatase inhibitors versus tamoxifen in early breast cancer: patient-level meta-analysis of the randomised trials. Lancet. 2015;386(10001): 1341-52.

2. Demicheli R, Ardoino I, Boracchi P, Coradini D, Agresti R, Ferraris C, et al. Recurrence and mortality according to estrogen receptor status for breast cancer patients undergoing conservative surgery. Ipsilateral breast tumour recurrence dynamics provides clues for tumour biology within the residual breast. BMC Cancer. 2010;10:656.

3. Pan HC, Gray R, Braybrooke J, Davies C, Taylor C, McGale P, et al. 20-Year risks of breast-cancer recurrence after stopping endocrine therapy at 5 years. N Engl J Med. 2017;377(19):1836-46.

4. Weigelt B, Glas AM, Wessels LF, Witteveen AT, Peterse JL, van't Veer LJ. Gene expression profiles of primary breast tumors maintained in distant metastases. Proc Natl Acad Sci U S A. 2003:100(26):15901-5.

5. Tang MH, Dahlgren M, Brueffer C, Tjitrowirjo T, Winter C, Chen $Y$, et al. Remarkable similarities of chromosomal rearrangements between primary human breast cancers and matched distant metastases as revealed by whole-genome sequencing. Oncotarget. 2015;6(35):37169-84.

6. Kroigard AB, Larsen MJ, Thomassen M, Kruse TA. Molecular concordance between primary breast cancer and matched metastases. Breast J. 2016; 22(4):420-30.

7. Demicheli R, Terenziani M, Bonadonna G. Estimate of tumor growth time for breast cancer local recurrences: rapid growth after wake-up? Breast Cancer Res Treat. 1998;51(2):133-7.

8. Uhr JW, Pantel K. Controversies in clinical cancer dormancy. Proc Natl Acad Sci U S A. 2011;108(30):12396-400.

9. Sosa MS, Bragado P, Aguirre-Ghiso JA. Mechanisms of disseminated cancer cell dormancy: an awakening field. Nat Rev Cancer. 2014;14(9):611-22.

10. Dittmer J. Mechanisms governing metastatic dormancy in breast cancer. Semin Cancer Biol. 2017;44:72-82

11. Selli C, Dixon JM, Sims AH. Accurate prediction of response to endocrine therapy in breast cancer patients: current and future biomarkers. Breast Cancer Res. 2016:18(1):118.

12. Clarke R, Tyson JJ, Dixon JM. Endocrine resistance in breast cancer-an overview and update. Mol Cell Endocrinol. 2015:418(Pt 3):220-34

13. Ma CX, Reinert T, Chmielewska I, Ellis MJ. Mechanisms of aromatase inhibitor resistance. Nat Rev Cancer. 2015:15(5):261-75.

14. Sims AH, Bartlett JMS. Approaches towards expression profiling the response to treatment. Breast Cancer Res. 2008;10(6):115.

15. Vendrell JA, Robertson KE, Ravel P, Bray SE, Bajard A, Purdie CA, et al. A candidate molecular signature associated with tamoxifen failure in primary breast cancer. Breast Cancer Res. 2008;10(5):R88

16. Turnbull AK, Arthur LM, Renshaw L, Larionov AA, Kay C, Dunbier AK, et al. Accurate prediction and validation of response to endocrine therapy in breast cancer. J Clin Oncol. 2015;33(20):2270-8.

17. Dunbier AK, Ghazoui Z, Anderson H, Salter J, Nerurkar A, Osin P, et al. Molecular profiling of aromatase inhibitor-treated postmenopausal breast tumors identifies immune-related correlates of resistance. Clin Cancer Res. 2013;19(10):2775-86

18. Patani N, Dunbier AK, Anderson $H$, Ghazoui Z, Ribas $R$, Anderson E, et al. Differences in the transcriptional response to fulvestrant and estrogen deprivation in ER-positive breast cancer. Clin Cancer Res. 2014; 20(15):3962-73.

19. Arthur LM, Turnbull AK, Webber VL, Larionov AA, Renshaw $L$, Kay $C$, et al. Molecular changes in lobular breast cancers in response to endocrine therapy. Cancer Res. 2014;74(19):5371-6.

20. Turnbull AK, Kitchen RR, Larionov AA, Renshaw L, Dixon JM, Sims AH. Direct integration of intensity-level data from Affymetrix and Illumina microarrays improves statistical power for robust reanalysis. BMC Med Genet. 2012;5:35.

21. Du P, Kibbe WA, Lin SM. lumi: a pipeline for processing Illumina microarray. Bioinformatics. 2008:24(13):1547-8.

22. Yu G, He QY. ReactomePA: an R/Bioconductor package for reactome pathway analysis and visualization. Mol BioSyst. 2016:12(2):477-9.

23. Hong FX, Breitling R, McEntee CW, Wittner BS, Nemhauser JL, Chory J. RankProd: a bioconductor package for detecting differentially expressed genes in meta-analysis. Bioinformatics. 2006;22(22):2825-7. 
24. Likhite N, Warawdekar UM. A unique method for isolation and solubilization of proteins after extraction of RNA from tumor tissue using trizol. J Biomol Tech. 2011;22(1):37-44.

25. Coleman O, Henry M, Clynes M, Meleady P. Filter-Aided Sample Preparation (FASP) for improved proteome analysis of recombinant Chinese hamster ovary cells. Methods Mol Biol. 2017;1603:187-94

26. Rappsilber J, Ishihama Y, Mann M. Stop and go extraction tips for matrixassisted laser desorption/ionization, nanoelectrospray, and LC/MS sample pretreatment in proteomics. Anal Chem. 2003;75(3):663-70.

27. Cox J, Mann M. MaxQuant enables high peptide identification rates, individualized p.p.b.-range mass accuracies and proteome-wide protein quantification. Nat Biotechnol. 2008;26(12):1367-72.

28. Vizcaino JA, Csordas A, Del-Toro N, Dianes JA, Griss J, Lavidas I, et al. 2016 update of the PRIDE database and its related tools. Nucleic Acids Res. 2016; 44(22):11033.

29. Haffner MC, Chaux A, Meeker AK, Esopi DM, Gerber J, Pellakuru LG, et al. Global 5-hydroxymethylcytosine content is significantly reduced in tissue stem/progenitor cell compartments and in human cancers. Oncotarget. 2011;2(8):627-37.

30. Perou CM, Sorlie T, Eisen MB, van de Rijn M, Jeffrey SS, Rees CA, et al. Molecular portraits of human breast tumours. Nature. 2000;406(6797): 747-52.

31. Nielsen TO, Parker JS, Leung S, Voduc D, Ebbert M, Vickery T, et al. A comparison of PAM50 intrinsic subtyping with immunohistochemistry and clinical prognostic factors in tamoxifen-treated estrogen receptor-positive breast cancer. Clin Cancer Res. 2010;16(21):5222-32.

32. Cochrane DR, Bernales S, Jacobsen BM, Cittelly DM, Howe EN, D'Amato NC, et al. Role of the androgen receptor in breast cancer and preclinical analysis of enzalutamide. Breast Cancer Res. 2014;16(1):R7.

33. Barbie DA, Tamayo P, Boehm JS, Kim SY, Moody SE, Dunn IF, et al. Systematic RNA interference reveals that oncogenic KRAS-driven cancers require TBK1. Nature. 2009;462(7269):108-U122.

34. Chen EY, Tan CM, Kou Y, Duan Q, Wang Z, Meirelles GV, et al. Enrichr: interactive and collaborative HTML5 gene list enrichment analysis tool. BMC Bioinformatics. 2013;14:128.

35. Ellis MJ, Suman VJ, Hoog J, Goncalves R, Sanati S, Creighton CJ, et al. Ki67 proliferation index as a tool for chemotherapy decisions during and after neoadjuvant aromatase inhibitor treatment of breast cancer: results from the American College of Surgeons Oncology group Z1031 trial (Alliance). J Clin Oncol. 2017;35(10):1061-9.

36. Jankowitz RC, Oesterreich S, Lee AV, Davidson NE. New strategies in metastatic hormone receptor-positive breast cancer: searching for biomarkers to tailor endocrine and other targeted therapies. Clin Cancer Res. 2017;23(5):1126-31.

37. Miller WR, Larionov A. Changes in expression of oestrogen regulated and proliferation genes with neoadjuvant treatment highlight heterogeneity of clinical resistance to the aromatase inhibitor, letrozole. Breast Cancer Res. 2010;12(4):R52.

38. Zhang XHF, Giuliano M, Trivedi MV, Schiff R, Osborne CK. Metastasis dormancy in estrogen receptor-positive breast cancer. Clin Cancer Res. 2013;19(23):6389-97.

39. Bartosh TJ. Cancer cell cannibalism and the SASP: ripples in the murky waters of tumor dormancy. Mol Cell Oncol. 2017;4(1):e1263715.

40. Barkan D, Green JE, Chambers AF. Extracellular matrix: a gatekeeper in the transition from dormancy to metastatic growth. Eur J Cancer. 2010;46(7): $1181-8$.

41. Wang G, Platt-Higgins A, Carroll J, de Silva RS, Winstanley J, Barraclough R, et al. Induction of metastasis by S100P in a rat mammary model and its association with poor survival of breast cancer patients. Cancer Res. 2006; 66(2):1199-207.

42. Prica F, Radon T, Cheng Y, Crnogorac-Jurcevic T. The life and works of S100P_from conception to cancer. Am J Cancer Res. 2016;6(2):562-76.

43. Yang RX, Stocker S, Schott S, Heil J, Marme F, Cuk K, et al. The association between breast cancer and S100P methylation in peripheral blood by multicenter case- control studies. Carcinogenesis. 2017;38(3):312-20.

44. Peng C, Chen H, Wallwiener M, Modugno C, Cuk K, Madhavan D, et al. Plasma S100P level as a novel prognostic marker of metastatic breast cancer. Breast Cancer Res Treat. 2016;157(2):329-38.

45. Dakhel S, Padilla L, Adan J, Masa M, Martinez JM, Roque L, et al. S100P antibody-mediated therapy as a new promising strategy for the treatment of pancreatic cancer. Oncogene. 2014;3:e92.
46. Basse $C$, Arock M. The increasing roles of epigenetics in breast cancer: implications for pathogenicity, biomarkers, prevention and treatment. Int J Cancer. 2015;137(12):2785-94.

47. Crea F, Nur Saidy NR, Collins CC, Wang Y. The epigenetic/noncoding origin of tumor dormancy. Trends Mol Med. 2015;21(4):206-11.

48. Fang F, Turcan S, Rimner A, Kaufman A, Giri D, Morris LG, et al. Breast cancer methylomes establish an epigenomic foundation for metastasis. Sci Transl Med. 2011;3(75):75ra25.

49. Tsang JS, Vencken S, Sharaf O, Leen E, Kay EW, McNamara DA, et al. Global DNA methylation is altered by neoadjuvant chemoradiotherapy in rectal cancer and may predict response to treatment-a pilot study. Eur J Surg Oncol. 2014;40(11):1459-66.

50. De Smet C, Loriot A. DNA hypomethylation in cancer: epigenetic scars of a neoplastic journey. Epigenetics. 2010;5(3):206-13.

51. Pathiraja TN, Xi Y, Lee AV, Santen R, Gannon F, Kaipparettu B, et al. Estrogen deprivation results in altered DNA methylation profile in breast cancer cells_role in endocrine resistance? Cancer Res. 2009;69(24):808s.

52. Harris ME, Bohni R, Schneiderman MH, Ramamurthy L, Schumperli D, Marzluff WF. Regulation of histone mRNA in the unperturbed cell cycle: evidence suggesting control at two posttranscriptional steps. Mol Cell Biol. 1991;11(5):2416-24.

53. Braunstein M, Liao L, Lyttle N, Lobo N, Taylor KJ, Krzyzanowski PM, et al. Downregulation of histone $\mathrm{H} 2 \mathrm{~A}$ and $\mathrm{H} 2 \mathrm{~B}$ pathways is associated with anthracycline sensitivity in breast cancer. Breast Cancer Res. 2016;18(1):16.

54. Zhu Z, Edwards RJ, Boobis AR. Increased expression of histone proteins during estrogen-mediated cell proliferation. Environ Health Perspect. 2009; 117(6):928-34

55. Sarkar S, Abujamra AL, Loew JE, Forman LW, Perrine SP, Faller DV. Histone deacetylase inhibitors reverse $\mathrm{CPG}$ methylation by regulating DNMT1 through ERK signaling. Anticancer Res. 2011;31(9):2723-32.

56. Lee JH, Choy ML, Ngo L, Foster SS, Marks PA. Histone deacetylase inhibitor induces DNA damage, which normal but not transformed cells can repair. Proc Natl Acad Sci U S A. 2010;107(33):14639-44.

57. Bolden JE, Shi W, Jankowski K, Kan CY, Cluse L, Martin BP, et al. HDAC inhibitors induce tumor-cell-selective pro-apoptotic transcriptional responses. Cell Death Dis. 2013;4:e519.

58. Ceccacci $\mathrm{E}$, Minucci S. Inhibition of histone deacetylases in cancer therapy: lessons from leukaemia. Br J Cancer. 2016;114(6):605-11.

59. Masri S, Lui K, Phung S, Ye J, Zhou D, Wang X, et al. Characterization of the weak estrogen receptor alpha agonistic activity of exemestane. Breast Cancer Res Treat. 2009;116(3):461-70.

60. Aguilar H, Sole X, Bonifaci N, Serra-Musach J, Islam A, Lopez-Bigas N, et al. Biological reprogramming in acquired resistance to endocrine therapy of breast cancer. Oncogene. 2010;29(45):6071-83.

61. Milosevic J, Klinge J, Borg AL, Foukakis T, Bergh J, Tobin NP. Clinical instability of breast cancer markers is reflected in long-term in vitro estrogen deprivation studies. BMC Cancer. 2013;13:473.

62. Simigdala N, Gao Q, Pancholi S, Roberg-Larsen H, Zvelebil M, Ribas R, et al. Cholesterol biosynthesis pathway as a novel mechanism of resistance to estrogen deprivation in estrogen receptor-positive breast cancer. Breast Cancer Res. 2016;18:58.

63. Coser KR, Wittner BS, Rosenthal NF, Collins SC, Melas A, Smith SL, et al. Antiestrogen-resistant subclones of MCF-7 human breast cancer cells are derived from a common monoclonal drug-resistant progenitor. Proc Natl Acad Sci U S A. 2009;106(34):14536-41.

64. Alves CL, Elias D, Lyng M, Bak M, Kirkegaard T, Lykkesfeldt AE, et al. High CDK6 protects cells from fulvestrant-mediated apoptosis and is a predictor of resistance to fulvestrant in estrogen receptor-positive metastatic breast cancer. Clin Cancer Res. 2016;22(22):5514-26. 